\title{
Scintillation cameras: A new clinical era has come
}

\author{
Raymond Taillefer, MD, FRCP, ABNM ${ }^{\mathrm{a}}$ \\ a Department of Nuclear Medicine, Hôpital du Haut-Richelieu, CISSS de la Montérégie Centre, \\ Saint-Jean-sur-Richelieu, Quebec, Canada
}

Received Apr 9, 2021; accepted Apr 9, 2021

doi: $10.1007 / \mathrm{s} 12350-021-02660-4$

\section{See related article, pp. 1933-1941}

Over the years, the major breakthroughs seen in nuclear cardiology, and in general nuclear medicine as well, have been intimately related to technological innovations in three different but closely linked together major scientific fields (1) Radionuclide imaging devices, (2) radiopharmaceuticals, and (3) image acquisition and analysis processes. Improvements in one of these three fields were almost always accompanied by new discoveries or new applications in the other two. Developments of new imaging devices may lead to new types of image analysis which in turn can stimulate the research for new radiotracers with different imaging characteristics and thus ultimately having a significant impact on clinical practice and patient management.

The Anger scintillation gamma camera has been the imaging device of choice in nuclear medicine and in nuclear cardiology for more than 60 years, replacing the rectilinear scanner. The basic concept of the Anger gamma camera design has not significantly changed in the last decades. A collimator projects an image of the distribution of the radionuclide onto a thin thallium activated sodium-iodide $[\mathrm{NaI}(\mathrm{Tl})]$ scintillation crystal. The addition of a trace amount of thallium as an activator is necessary since pure sodium iodide produces very little scintillation after interaction of gamma radiations at room temperature. The light emitted in the crystal travels in all directions and is proportional to the

Reprint requests: Raymond Taillefer, MD, FRCP, ABNM, Department of Nuclear Medicine, Hôpital du Haut-Richelieu, CISSS de la Montérégie Centre, 920 Boulevard du Séminaire Nord, Saint-Jeansur-Richelieu, Quebec J3A 1B7, Canada; rtaillefer@hotmail.com J Nucl Cardiol 2022;29:1942-5.

1071-3581/ $\$ 34.00$

Copyright (c) 2021 American Society of Nuclear Cardiology. energy absorbed. An array of photomultipliers tubes detects it and converts the light distribution into a set of electronic signals.

The Anger gamma camera is particularly well adapted for imaging the $140 \mathrm{keV}$ gamma rays emitted by ${ }^{99 \mathrm{~m}}$ Tc-technetium, the most frequently used monophotonic radionuclide in nuclear cardiology. Since its first description by Hal Anger in 1958, ${ }^{1}$ this type of imaging device has been continuously perfected over the years. Addition of digital processing to the analog detector electronics allowed significant improvements in count rate capability, uniformity, and intrinsic spatial resolution. The clinical usefulness of scintillation cameras was extended by adding two or more detectors to create single photon computed tomography (SPECT) with multiple rotating gamma cameras, thus further improving the overall sensitivity and resolution. The relatively low cost and easy installation of SPECT with the sodium iodide crystal scintillation detector camera (in comparison to a positron emission tomography imaging facility) makes it the most widely available nuclear cardiology imaging device in the world still today. But these statistics are likely to change in the very near future with the newly available large field-of-view scintillation camera equipped with solid-state semiconductor detectors made of cadmium-zinc-telluride $(\mathrm{CZT}) .^{2-4}$

Although the Anger gamma cameras are still widely used, it shows important limitations. $\mathrm{NaI}(\mathrm{Tl})$ crystals are hygroscopic and fragile. They must be handled with care and room temperature should not be changed abruptly because sudden changes in temperature can cause cracks in the crystal. The fundamental performance limitations imposed by the collimator and the detection systems restrict the intrinsic spatial resolution to $3-4 \mathrm{~mm}$. The energy resolution is also relatively poor, affecting both the intrinsic spatial resolution and the intrinsic efficiency. A significant fraction of scattered photons is contributing to decrease the image contrast. The design of the gamma cameras is also limited. The use of 
collimators, crystal, photomultipliers tubes and electronic circuits makes them rather bulky and the shielding needed to reject scattered radiation makes them rather heavy or immobile. Therefore, it is not surprising that continuous researches have been made with the goal to obtain a better imaging device showing improved imaging characteristics.

Various types of crystals (solid-state detectors with crystals such as cesium iodide or germanium and silicon) have been proposed to replace sodium iodide. CZT has emerged as one of the most interesting and promising in that purpose. CZT detectors are semiconductors made of cadmium, zinc, and tellurium metals. It provides very high efficiency for gamma ray detection because of their high atomic numbers. This high detection efficiency allows the CZT detectors to be made as small as $2 \mathrm{~mm}$ thick with almost $100 \%$ efficiency for the detection of $100 \mathrm{keV}$ photons. Furthermore, the energy resolution of these detectors is very good (approximately $6 \%$ ) for a wide range of gamma ray energies. They can be operated at room temperature and the electronics used are very similar to those of other conventional types of scintillation cameras. CZT detectors improve the energy, spatial, and contrast resolution through the use of direct conversion (in comparison to indirect conversion with conventional $\mathrm{NaI}$ gamma camera) of the energy and location of the detected photon into an electronic pulse (true digital camera).

Many manufacturers in the late 2000s developed innovative designs of dedicated cardiac cameras, especially with CZT solid-state detectors. ${ }^{5-7}$ These cameras had the potential for up to 10-fold increase in count sensitivity with even a slight gain in resolution. These significantly improved imaging characteristics opened the field of nuclear cardiology to the development of entirely new imaging protocols. The significant gain in sensitivity (much higher counting statistics) can be used to either decrease the overall image acquisition time, decrease the injected dose to reduce the patient's exposure to radiation, or an hybrid protocol decreasing both the acquisition time and the dose to a certain level.

Decreasing the acquisition time has been a major goal in nuclear cardiology in order to enhance patient comfort, decrease the likelihood of patient motion artifacts during image acquisition, improve patient throughput especially in a very busy nuclear cardiology department, and be competitive to other cardiac imaging modalities which can be performed faster. Some studies have showned that a stress or rest ${ }^{99 \mathrm{~m}} \mathrm{Tc}$-labeled radiotracer myocardial perfusion imaging (MPI) study could be completed within 2-3 minutes. ${ }^{7}$

Using the same dose and the same imaging time with the new CZT cameras can also be very beneficial to improve the image quality and ultimately the diagnosis of coronary artery disease (CAD) in very obese patients. Higher count sensitivity can also be used to perform respiratory gating, ${ }^{8}$ helping to reduce the negative effect of chest motion on the image and the artifacts related to the presence of various ${ }^{99 \mathrm{~m}} \mathrm{Tc}$-labeled radionuclides in the liver and subdiaphragmatic bowel loops. Because of the relatively small size of the detectors, cameras design can be quite flexible and easily adapted to nuclear cardiologic procedures, enabling more ergonomic design and increasing patients comfort during the image acquisition. The higher count efficiency also could allow to perform dynamic SPECT tracers acquisition and «true» stress acquisitions of myocardial function and perfusion simultaneously. One example is the use of ${ }^{99 \mathrm{~m}}$ Tc-teboroxime or related radiotracers ${ }^{9}$ which imposed significant technical challenges with a very rapid myocardial uptake and washout (myocardial halflife of less than 10 minutes). Having access to new highly sensitive and dynamic SPECT cameras certainly would promote a renewed interest in such radiotracers which were quickly abandoned in clinical practice because of the imaging device limitations at the time they have been approved.

Radiation exposure from medical procedures has become a major concern in several countries. Therefore, having a very sensitive imaging device, it is now possible to use the same imaging time than the one used with conventional gamma cameras but to decrease the injected dose of radionuclide by a significant factor, without modifying the overall diagnostic accuracy.

The first commercially available cardiac-dedicated SPECT camera using CZT detectors was the D-SPECT manufactured by Spectrum Dynamics (Israel). This was a small system with nine detectors. ${ }^{5}$ Since then, other systems have been introduced in clinical practice such as Discovery NM 530C from GE Healthcare ${ }^{10}$ or the Cardius 3XPO from Digirad, using thallium activated cesium iodide crystal. ${ }^{2,11}$ Those systems were using relatively small field-of-view detectors due to the cost and more limited technology. However, significant progress have recently been made and it is now possible (GE Healthcare NM/CT $870 \mathrm{CZT}$ model) to have a large field-of-view CZT rectangular digital detectors consisting of 130 CZT modules of $39.4 \times 39.4 \times 7.25 \mathrm{~mm}$ each, with a field-of-view of $39.36 \mathrm{~cm} \times 51.17 \mathrm{~cm}$. Every time a new technology emerges, it is obviously important to compare its results to those obtained with its predecessor. New technological benefits have to be translated into improvements in overall diagnostic accuracy. With the increasing clinical availability of the semi-conductors CZT cameras, more results have been published. In a meta-analysis by Cantoni et al, ${ }^{12}$ obtained from 40 articles looking at the diagnostic performance of MPI with conventional and CZT SPECT 
imaging, both systems showed a good diagnostic performance in detection of angiographic proven CAD, with a slightly higher accuracy for CZT-SPECT: a pooled sensitivity and specificity of $85 \%$ and $66 \%$ for conventional SPECT and $89 \%$ and $69 \%$ respectively for CZT-SPECT. Head-to-head comparative studies are rather rare in this new field of investigation. Neill et $\mathrm{al}^{13}$ reported a sensitivity, a specificity and accuracy of $84 \%$, $50 \%$, and $76 \%$ for conventional SPECT vs $92 \%, 83 \%$, and $90 \%$ respectively for CZT-SPECT. Gimelli et al ${ }^{14}$ also demonstrated that CZT-SPECT was superior to conventional SPECT for detection of global and regional ischemia.

In the current issue of the Journal, Bonnefoy et $\mathrm{al}^{15}$ report the results of a prospective study using a novel whole-body CZT SPECT/CT (DNM 670 CZT from GE Healthcare) with the goal of determining the equivalence between conventional image acquisition protocol and reduced time protocol for myocardial perfusion imaging (MPI) performed with ${ }^{201}$ Thallium. The stress images were realized in list mode in 103 consecutive patients. Data were reconstructed using list mode acquisition to simulate $75 \%$ and $50 \%$ of total count statistics, therefore mimicking a reduction of $25 \%$ and $50 \%$ of the full original acquisition time, respectively. They reported an equivalence in the number of myocardial segments showing lower than $70 \%$ of the maximal intensity and functional parameters such as ejection fraction, end-diastolic, and end-systolic volumes between the three protocols up to a reduction of $50 \%$ of counts. This study shows that the imaging time can be reduced by half the conventional one without compromising the overall quality of the resultant data. It will be interesting to have more results on the diagnostic accuracy since only stress images have been analyzed. However, this represents an important step in nuclear cardiology. Effectively, several nuclear medicine laboratories over the world are performing MPI studies in conjunction with nuclear cardiologists, cardiologists, and radiologists. It is likely that those laboratories will strongly consider acquiring these types of new wholebody CZT SPECT/CT cameras given their improved image characteristics, the versatility of image acquisition protocols and the possibility of performing real whole-body studies, increasing the number and the types of studies that can be done in a day. This can also benefit to very obese patients or in some specific nuclear cardiologic procedures such as ${ }^{99 \mathrm{~m}} \mathrm{Tc}$-pyrophosphate imaging in patients with cardiac amyloidosis where a whole-body study can help to assess other sites of muscular involvement. It could also be beneficial to studies involving ${ }^{111}$ In-white blood cells imaging where cardiac involvement is suspected.
There is no doubt that we are entering into a new clinical era where it is likely that conventional $\mathrm{NaI}$ crystal SPECT cameras will be progressively but irremediably replaced by CZT SPECT/CT cameras, giving the field on nuclear cardiology access to a wide variety of imaging protocols which can be tailored to the specific clinical needs. As appropriately stated by Karimeddini and Bergmann in their editorial published in $2015^{16}$ on the new SPECT cameras using solid-state CZT detectors, "the future of nuclear cardiology is solid (state) and bright".

\section{Disclosure}

Raymond Taillefer declares that he has no conflict of interest.

\section{References}

1. Anger HO. Scintillation camera with multichannel collimators. J Nucl Med 1964;5:515-31.

2. Maddahi J, Mendez R, Mahmarian J, et al. Prospective multicenter evaluation of rapid gated SPECT myocardial perfusion upright imaging. J Nucl Cardiol 2009;16:351-7.

3. Ben-Haim S, Hutton BF, Van Grantberg D. Simultaneous dualradionuclide myocardial perfusion imaging with a solid-state dedicated gamma-camera. Eur $\mathrm{J}$ Nucl Med Mol Imaging 2010;37:1710-21.

4. Esteves FP, Raggi P, Folks RD, et al. Novel solid-state-detector dedicated cardiac camera for fast myocardial perfusion imaging: Multicenter comparison with standard dual detector cameras. J Nucl Cardiol 2009;16:927-34.

5. Sharir T, Ben-Haim S, Merzon K, et al. High-speed myocardial perfusion imaging: Initial clinical comparison with conventional dual detector Anger camera imaging. J Am Coll Cardiol Imaging 2008;1:156-63.

6. Berman DS, Kang X, Tamarappoo B, et al. Stress thallium-201/ rest technetium-99m sequential dual isotope high-speed myocardial perfusion imaging. J Am Coll Cardiol Imaging 2009;2:27382.

7. Sharir T, Slomka PJ, Hayes SW, et al. Multicenter trial of highspeed versus conventional single-photon emission computed tomography imaging: Quantitative results of myocardial perfusion and left ventricular function. J Am Coll Cardiol 2010;55:1965-74.

8. Buechel RR, Pazhenkottil AP, Katz R, et al. Nuclear myocardial perfusion imaging with a cadmium-zinc-telluride detector technique: Optimized protocol for scan time reduction. J Nucl Med 2010;51:46-51.

9. Taillefer R. ${ }^{99 \mathrm{~m}} \mathrm{Tc}$-labeled boronic acid derivatives for SPECT myocardial perfusion imaging: Improved version 2.0. J Nucl Cardiol 2020. https://doi.org/10.1007/s12350-020-02137-w.

10. Buechel RR, Herzog BA, Husmann L, et al. Ultrafast nuclear myocardial perfusion imaging on a new gamma camera with semiconductor technique: First clinical validation. Eur J Nucl Med Mol Imaging 2010;37:773-8.

11. Bai C, Conwell R, Kindem J, et al. Phantom evaluation of a cardiac SPECT/CT system that uses a common set of solid-state detectors for both emission and transmission scans. J Nucl Cardiol 2010;17:459-69.

12. Cantoni V, Green R, Acampa W, et al. Diagnostic performance of myocardial perfusion imaging with conventional and CZT single- 
photon computed tomography in detecting coronary artery disease: A meta-analysis. J Nucl Cardiol 2019. https://doi.org/10.1007/s1 2350-019-01747-3.

13. Neill J, Prvrulovich EM, Fish MB, et al. Initial multicenter experience of high-speed myocardial perfusion imaging: Comparison between high-speed and conventional single-photon emission computed tomography with angiographic validation. Eur J Nucl Med Mol Imaging 2013;40:1084-94.

14. Gimelli A, Bottai M, Giorgetti A, et al. Comparison between ultrafast and standard SPECT in patients with coronary artery disease: A pilot study. Circ Cardiovasc Imaging 2011;4:51-8.
15. Bonnefoy PB, Janvier L, Arede C, et al. Reduced acquisition time for thallium myocardial perfusion imaging with large field cadmium-zinc-telluride SPECT/CT cameras: An equivalence study. J Nucl Cardiol. https://doi.org/10.1007/s12350-021-02611-z.

16. Karimeddini D, Bergmann S. The state of the future is solid. J Nucl Cardiol 2015. https://doi.org/10.1007/s12350-015-0224-2.

Publisher's Note Springer Nature remains neutral with regard to jurisdictional claims in published maps and institutional affiliations. 